\title{
A NOVEL EXPERIMENTAL DESIGN OF A REAL-TIME VR TRACKING DEVICE
}

\author{
Barhoush, Yazan A M (1); \\ Nanjappan, Vijayakumar (1); \\ Thiel, Felix (2); \\ Georgiev, Georgi V. (1); \\ Swapp, David (2); \\ Loudon, Brian (3) \\ 1: University of Oulu, Finland; \\ 2: University College London, UK; \\ 3: Loud1Design Ltd., Glasgow, UK
}

\begin{abstract}
Virtual Reality (VR) is progressively adopted at different stages of design and product development. Consequently, evolving interaction requirements in engineering design and development for VR are essential for technology adoption. One of these requirements is real-time positional tracking. This paper aims to present an experimental design of a new real-time positional tracking device (tracker), that is more compact than the existing solution, while addressing factors such as wearability and connectivity. We compare the simulation of the proposed device and the existing solution, discuss the results, and the limitations. The new experimental shape of the device is tailored towards research, allowing the engineering designer to take advantage of a new tracker alternative in new ways, and opens the door to new VR applications in research and product development.
\end{abstract}

Keywords: Virtual reality, Simulation, Virtual Engineering (VE), Motion Tracking, Positional Tracking

\section{Contact:}

Barhoush, Yazan A M

University of Oulu

Center for Ubiquitous Computing

Finland

yazan.barhoush@oulu.fi 


\section{INTRODUCTION}

Various current design methods and tools rely on technologies that enable new opportunities and possibilities in product development. Virtual Reality (VR) technology gains wider adoption at different stages of engineering design and product development (Carulli et al., 2018; Horvat et al., 2019; Gerschütz et al., 2019). Consequently, evolving interaction requirements in design and product development for VR are essential for the adoption, especially in the case of engineering design applications. One of these requirements is real-time positional tracking, where applications require an accurate sampling of the spatial environment of the user to sustain the illusion of control (Honnet and Lopes, 2019). Compact, precise, and wearable devices are essential to match increasingly complex interaction requirements of VR systems utilized on different stages of in engineering design. Such requirements are valid for all design stages (Gerschütz et al., 2019). In this paper we focus on motion tracking devices for VR.

\subsection{Current state-of-the-art in motion tracking for VR}

Motion capture systems are the current state-of-the-art for recording the movements of people and objects (Kruk and Reijne, 2018). However, when it comes to VR systems, the current industry standards for motion capture, such as VICON ${ }^{1}$ and OptiTrack ${ }^{2}$, require multiple specialized cameras or additional accessories, which makes these systems inaccessibly expensive and bulky.

One alternative, for tracking small and fast movements, are Inertial Measurement Units (IMUs). However, IMUs do not measure "absolute position", which makes them, when used alone in VR, insufficient for precise and continuous tracking of natural movements; a phenomenon referred to as "drift" (Wittmann et al., 2019). Another option is Microsoft's Kinect, a consumer level 3D motion-sensing camera device, designed to track the movements of users facing a video game display, which uses depth information to draw real-time pose (position and orientation) of a human body. However, a single Kinect requires the body to be located in front of the camera and thus, cannot track motion in 360 degrees (Capece et al., 2018). Some motion tracking systems in research, combine both Kinect and IMUs to compensate for the limitations of using each separately, but the tracking accuracy is still unacceptable or questionable for some application, such as rehabilitation (Naeemabadi et al., 2018; Du et al., 2018; Tian et al., 2015).

Due to the recent rise in popularity within the consumer market, there has been an increased demand for a more accessible and cost-effective solution. The majority of these designs are aimed at hand-held controller tracking, either by utilizing Inside-out computer vision based simultaneous localization and mapping (SLAM) or Outside-out Base Station and laser sensor approaches.

Inside-out tracking can be found on products, such as the Oculus Quest and Pico. The camera is located on the device being tracked and looks out to determine how its position is changing in relation to the external environment. As the headset moves, the sensor re-coordinates its place in the room, creating the illusion of moving through the virtual environment in real time.

Inside-out allows for mobility, since it does not restrict the "play" space, and can be done with or without markers around the environment. However, accuracy and latency remain a problem for now, since Inside-out tracking employs "good" computer vision while requiring all the computations to be done by the headset itself.

A further limitation is that objects can become hidden from the tracking camera when they are behind other objects, a technical problem known as occlusion. In the case of Inside-out, this can be the player's body, for example, when the hands are behind the back, close to sides or close to headset.

Oculus Rift, HTC Vive and PlayStation (PS) VR are examples where Outside-in tracking can be seen in action. The headset and controllers are tracked by an external device. In the case of the Vive, it is the Base Station. For the Oculus Rift, the Oculus Sensor is placed to point at the headset. The PS VR has a camera with similar setup to the Oculus Rift. In all of these cases, the Base Station or sensor(s) are placed in the room and determine the position of the headset in relation to its environment.

Outside-in solutions remain fixed and comparably -to the Inside-in- better when it comes to accuracy and latency. For example, the Vive allows for placing more Base Stations around the room to improve accuracy and latency.

${ }^{1}$ Location Based VR by Vicon. https://www.vicon.com/applications/location-based-virtual-reality

${ }^{2}$ Motion Capture for VR by OptiTrack. http://optitrack.com/applications/virtual-reality/index.html 
Although Outside-in solutions seem to be the most viable solutions in the market for research, they also suffer from occlusion due to the controller's geometry or the placement of markers on it (Suznjevic et al., 2017).

\subsection{Motion Tracking with The Vive Tracker}

The Vive system allows for the use of the Vive $\operatorname{Tracker}^{3}$ (a separate motion tracking device that can be attached to objects, so that they can be tracked) with its Outside-in Tracking. A Vive Tracker can be used to enable tracking and transmission of specific data. It can be a good development kit ${ }^{4}$ for developing VR tracked objects and controllers; it allows for inexpensive tracking solution for objects. Manufacturers of accessories do not need to develop a tracking solution if they can simply mount the Tracker on it. Therefore, allows to start rethinking the entire applications to object motion tracking. Further, we review selected works which utilize the Vive Tracker in research, categorizing them according to the field of application and describe the design, as well as the application for each work.

\subsection{Motivation for an Experimental Tracker Design}

The aim of this paper is to present an experimental tracker design, that is more compact than the original, while addressing factors such as wearability and connectivity. The following subsections discuss example applications that benefit hugely from having a greater access to precise movement tracking using the Vive Tracker. The new experimental shape of the design is tailored towards research; where it could allow the highlighted applications to take advantage of a novel tracker alternative in new ways, and opens the door to a new VR applications in research and engineering product development.

\subsubsection{Applications in Medicine and Rehabilitation}

A proof-of-concept VR controller, for rehabilitation of users with limited physical mobility, was realized by Barhoush et al. (2020). Their implementation utilized the Vive Tracker in an experiment to investigate the usability of the system and the effectiveness and reliability of the controller in an empathic setup.

In another study, Burdea et al. (2019) presented a design to enable a caging-based natural grasp in VR, and integrated it into an assistive system for post-stroke tele-rehabilitation. Their design used the Vive Tracker and demonstrated that the system captures fine-grained hand motions.

\subsubsection{Applications in Wearable and Haptics}

The Vive Tracker can also be found on wearables. Tinguy et al. (2020) prototyped a wearable actuated tangibles, where the Vive Tracker is attached on the external side. The prototype is composed of a 3D-printed structure to placed on the back of the hand. The side of the 3D-printed structure housed an actuator which controls the motion of a rigid link holding the tangible object. By moving the rigid link, the motor brings the tangible object towards or away from the user's palm. Another Vive Tracker wearable use is mentioned by Freire et al. (2020). They described an e-textile glove. The glove is designed to facilitate research scientists and students carrying out detailed and complex manipulation of simulated 3d molecular objects in VR.

Haptic applications can be used to improve VR immersion on a level further than simply audio and visual. In attempt to provide realistic haptic feedback for games utilizing projectile launching weapons, Rahimi et al. (2020) designed and implemented a VR gun, which has recoil adjustability (haptic feedback system) for the Vive Tracker. In a different haptic implementation, an interchangeable wheel design was used to provide different sensations. It was developed to render fingertip haptics when interacting with virtual surfaces (Whitmire et al., 2018).

\subsubsection{Applications in Handheld Controllers}

In the area of handheld applications, shape-shifting as well as grasp sensation rendering controllers are another area of application where the Tracker has been used as a mean for VR tracking. Shigeyama et al. (2019) proposed a weight Shifting hand-held VR controller, that rendered a 2D shape, by changing

\footnotetext{
${ }^{3}$ VIVE ${ }^{\mathrm{TM}}$ Tracker. https://www.vive.com/eu/accessory/vive-tracker/

${ }^{4}$ VIVE $^{\mathrm{TM}}$ Tracker For Developers. https://developer.vive.com/eu/vive-tracker-for-developer/
} 
its mass properties on a 2D planar area. Another handheld controller that uses weight shifting was developed by (Zenner et al., 2020). It leveraged the airflow that occurs at the controller during interaction to provide a range of different haptic sensations; it can increase or decrease its surface area and by this also adapts its mass distribution. In another study, Murray et al. (2018) presented a variable shape and variable stiffness controller handle for use in VR. The controller handle transitions between two static states: a semi-rigid, large diameter state when pressurized and a soft, compressible, smaller diameter state when depressurized.

When it comes to grasp sensations rendering, Sinclair et al. (2019) developed a controller that renders touch and grasp haptic sensations in VR. It could supports human-scale forces during touch and grasp, resist the user's grasp force and exhibit variable stiffness and compliance. Another hand-held controller, that leverages grasp as an additional modality for VR was realized by Yi et al. (2019). The pressuresensitive surface of the controller was designed to recognize two different grasp-poses (precision grip and power grip) and detect grasp-force.

\section{PROPOSED TRACKED OBJECT DESIGN}

In this section we describe the requirements, design and rationale of the new tracked object (tracker) design we propose. The Valve Tracking system, shown in Figure 1, is comprised of Base Stations, Headmounted Displays (HMDs), controllers (e.g., The Vive Tracker) and a computer. HMDs and controllers are examples of tracked objects. The Base Stations emit Infrared (IR) reference signals (periodically sweeping the space with a laser at a fixed frequency), timestamped by tracked objects (photo-diodes placed in tracked objects can detect these sweeps). Timestamps are then used to triangulate position (either using information embedded in modulated light at the beam, or synchronization pulses, can reconstruct the exact angle at which the laser hit the object). Thus, tracked object positioning can be accurately retrieved.

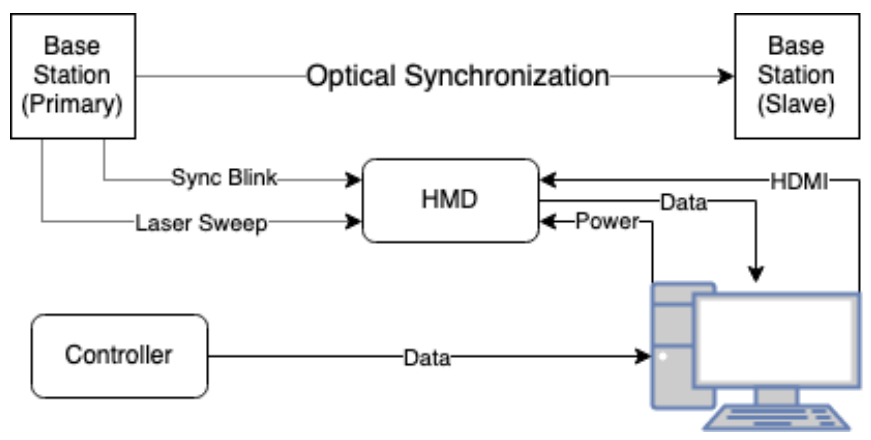

Figure 1. Valve tracking system

\subsection{Sensor Placement Requirements}

According to tracked object design guidelines, provided by the SteamVR Tracking Hardware Development Kit $(\mathrm{HDK})^{5}$, objects that track well have geometries designed for optimal sensor placement, where:

1. Four sensors need to be visible (minimum); taking into consideration that one must be $8 \mathrm{~mm}$ out of the plane-sensors have $\mathrm{a} \pm 60^{\circ}$ viewing angle.

2. The distance between sensors needs to be maximized.

3. A baseline needs to be created in three axes.

\subsection{Proposed Design and its Rationale}

When taking into consideration the sensor placement requirements, we propose a design that satisfies the requirements above. The design rationale is to minimize sensor occlusion (a main limitations of Outside-in tracking) by having constant negative-Gaussian curvature surface of revolution generated by a tractrix about its asymptote. Such surface is known as a pseudosphere (Stevenson, 2019).

\footnotetext{
${ }^{5}$ Welcome to Steamworks. https://partner.steamgames.com/vrlicensing
} 
To produce a pseudosphere ${ }^{6}$, the following parameterisation was used in order to get a more uniform mesh:

$$
\begin{aligned}
& d=a \tan (-a)+\frac{\pi}{2}, w=a \tan \left(\frac{2 a \times v}{\pi-a}\right)+\frac{\pi}{2}, v 1=\frac{(\pi-2 b)(w-d)}{(\pi-2 d)}+b \\
& f(u, v 1)=\left(\cos (u) \sin (v 1), \sin (u) \sin (v 1), \cos (v 1)+\log \left(\tan \left(\frac{v 1}{2}\right)\right)\right.
\end{aligned}
$$

Where $a$ controls how much the mesh is compressed or stretched, and $b$ cuts off the peaks of the pseudosphere ( $b \leq v 1 \leq \pi-b$, where $b$ can be chosen arbitrarily), otherwise the pseudosphere goes to infinity at both ends.

To improve baselines along three axes, two pseudospheres where used instead of one. To maximize the distance between the sensors, we experimented with the scale of the shape. Figure 2 shows the outcome when the whole thing is modelled in OpenScad.

\subsection{Employed Design Evaluation Tools}

The SteamVR HDK includes a software (HMD Designer) for product development. Since a tracked object performance is determined by its shape, the software allows for validating design decisions to reduce risk through simulation. The HMD Designer takes STL files as input for objects and obstacles and generates sensor placement with up to 32 sensors; produces SCAD models showing sensor placement; simulates sensor placement and outputs results.

\section{RESULTS AND DISCUSSION}

We feed the Vive Tracker model as well as our own model to the HMD Designer and compare simulation results. An obstacle was also modelled suggesting where sensors cannot be placed for a docking mechanism ${ }^{7}$.

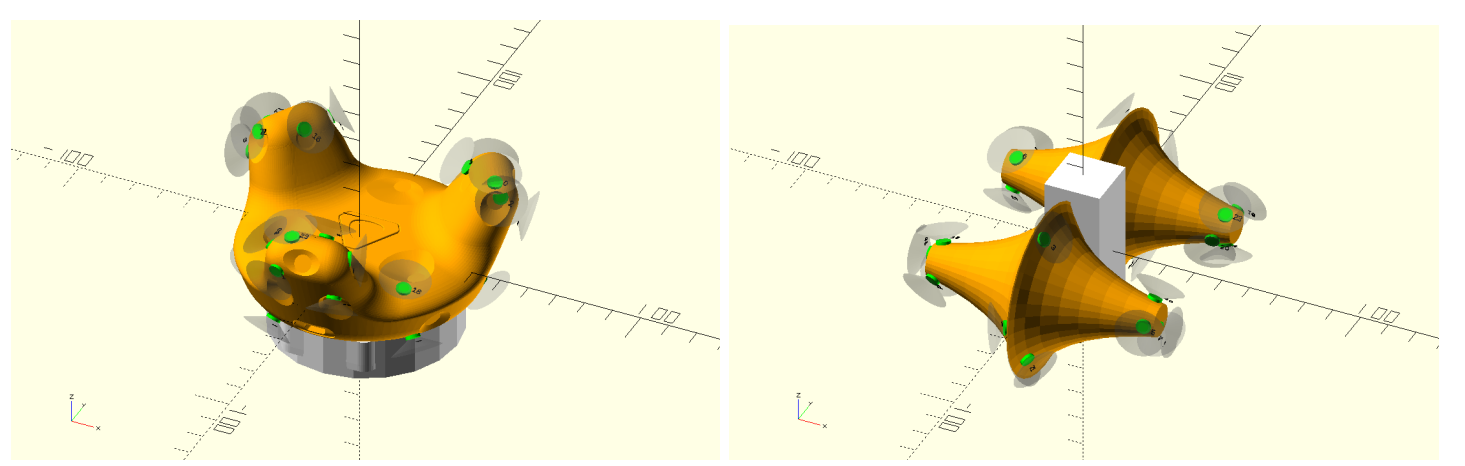

Figure 2. The Vive Tracker (Left) and our proposed model (Right), with sensors placed. Obstacle is shown in white, model is in yellow and sensors in green

When comparing the models' volumes, our model is $20 \%$ smaller. Moreover, our model allows for more wearability options due its various mounting orientations; the Tracker, for example, can be mounted from one side while our model is orientation agnostic (it can be mounted from the top or the bottom). This also leads to a better connectivity (interface exposure) when connecting an accessory.

\subsection{Simulation Score}

The simulation score is an indicator to the "quality" of a model. The lower the score, the better. The total number of simulated sensors was 24 (the same number of sensors in Vive controllers). The simulation quality was set to max, with no normal shifts. The number of permutations was 12 . The final quality score for the Vive Tracker was 65.617/1000, while our own model was 45.540/1000.

\footnotetext{
${ }^{6}$ Equations for the Pseudosphere. http://www.geom.uiuc.edu/zoo/diffgeom/pseudosphere/eqns.html

7 Tracker Developer Resources. https://developer.vive.com/resources/vive-sense/hardware-guide/
} 


\subsection{Simulation plots}

The HDK Design Software outputs 2D and 3D simulation plots. The 2D plots, produced by the Sensor Simulation Plotter, are useful for comparing results at a glance and highlighting potential shortcomings of the design. The 3D plots (spherically wrapped visualizations of the 2D plots surrounding the object being simulated), produced by the HMD Designer Viewer, are useful for pinpointing poses that exhibit low performance when "lining up" the Point of View (POV) marker with potential problem areas. The first plot, shown in Figure 3, is the 2D Number of Visible Sensors. It is an initial indicator of sensor placement quality, where 4 sensors are required to initiate tracking and 5 sensors are preferred.
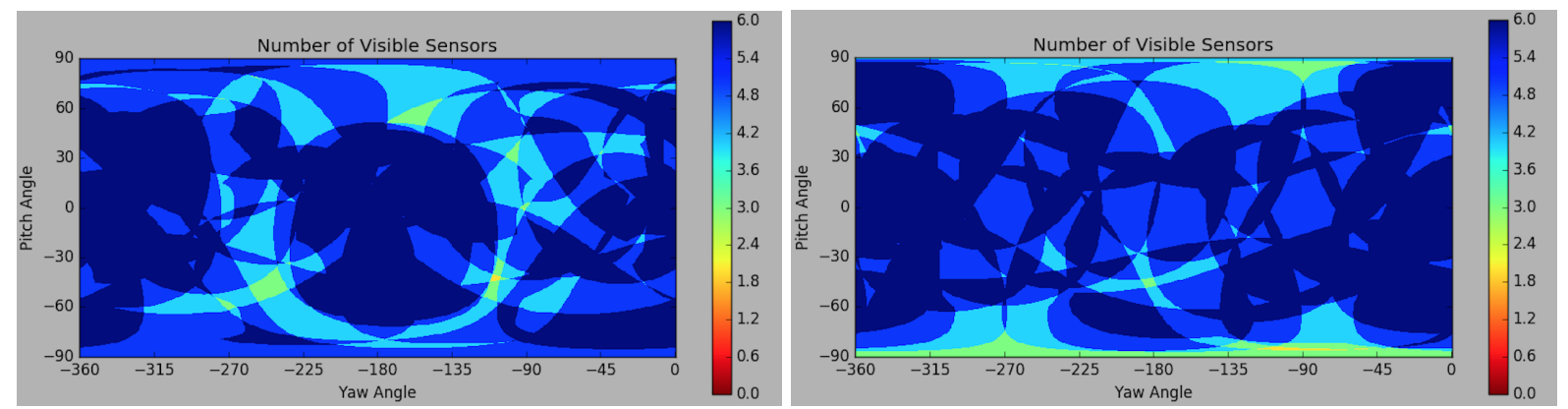

Figure 3. 2D Number of Visible Sensors for the Vive Tracker (Left) and our model (Right)

Its 3D wrapped visualization, the 3D Number of Visible Sensors view in Figure 4, shows the number of sensors that can be seen by the Base Stations at a given surface point of the object; increasing the number of visible sensors increases the number of "data points" available for the Base Stations.
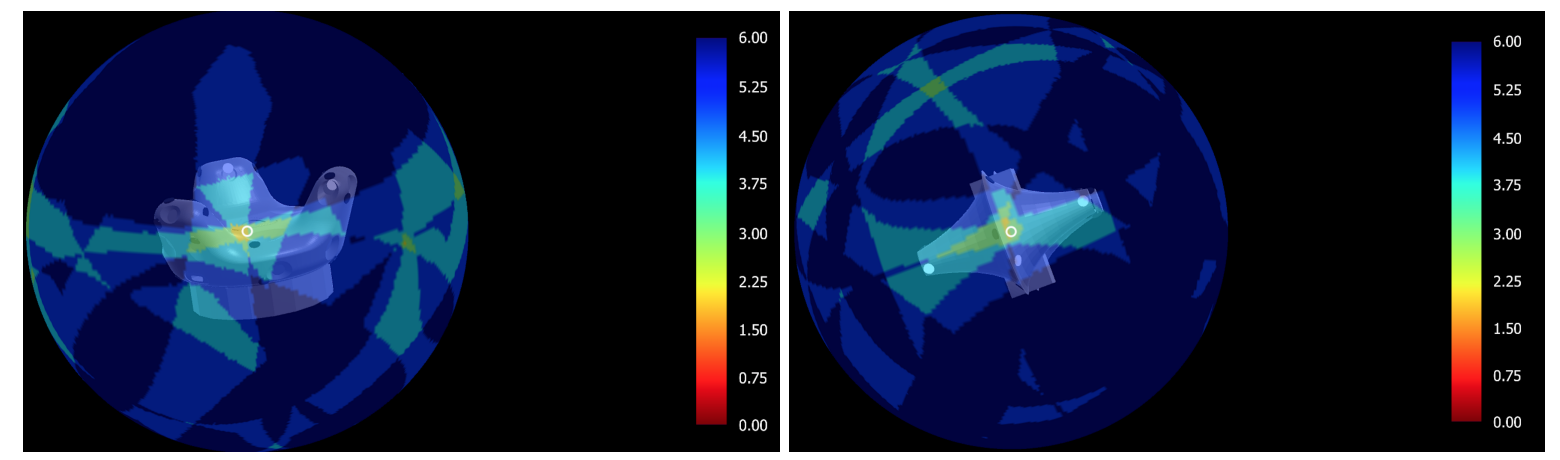

Figure 4. 3D Number of Visible Sensors for the Vive Tracker (Left) and our model (Right)

The second plot, shown in Figure 5, is the 2D Pose Rotation Error. It highlights object's sides which drive rotation errors. It is used to ensure that sensors are placed outside of the plane which other coplaner sensors create.
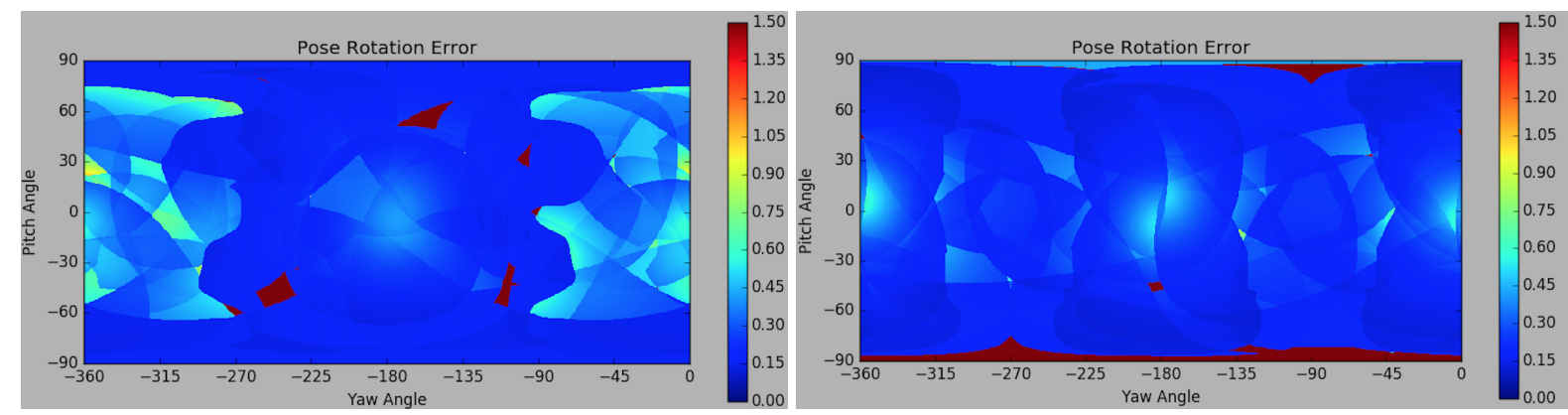

Figure 5. 2D Pose Rotation Error for the Vive Tracker (Left) and our model (Right) 
Its wrapped visualization, the $3 D$ Pose Rotation Error view, is shown in Figure 6. It demonstrates how co-planer sensors lead to a rotation error and the benefits of a baseline in three axes. Using it, the changes in the relationship between highlighted sensors can be observed when rotating the object.
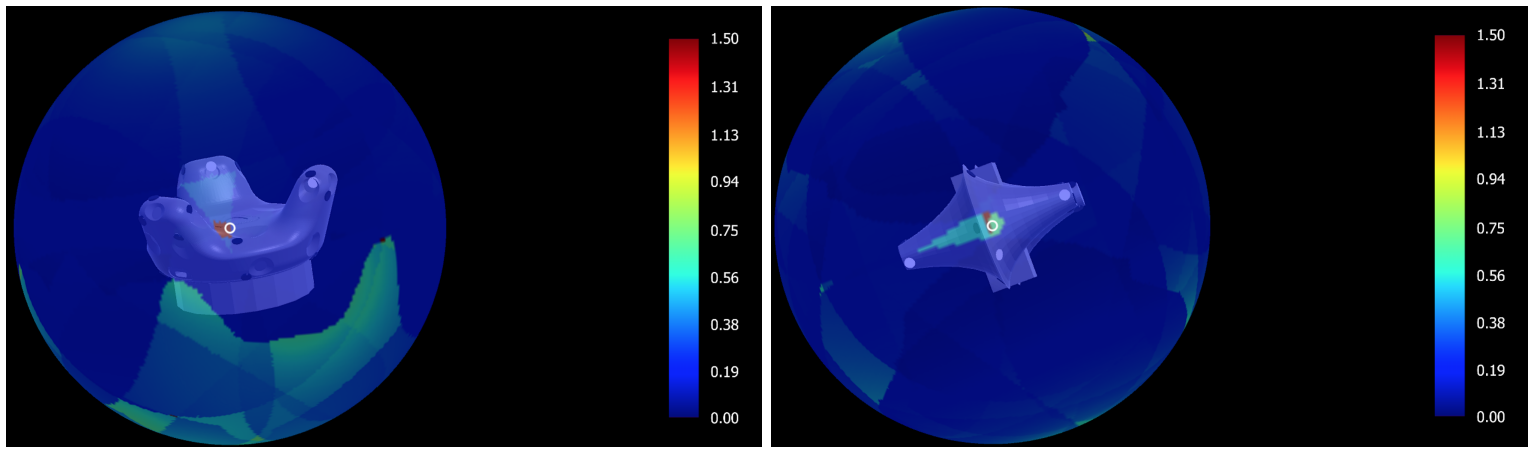

Figure 6. 3D Pose Rotation Error for the Vive Tracker (Left) and our model (Right)

The third plot, shown in Figure 7, is the $2 D$ Initial Pose Possible. It shows the requirements for initiating tracking. It resembles the Number of Visible Sensors plot and shows whether an object initiates tracking or not from a given pose. Its discrete colors, compared to the other plots, come from hard limits on requirements.
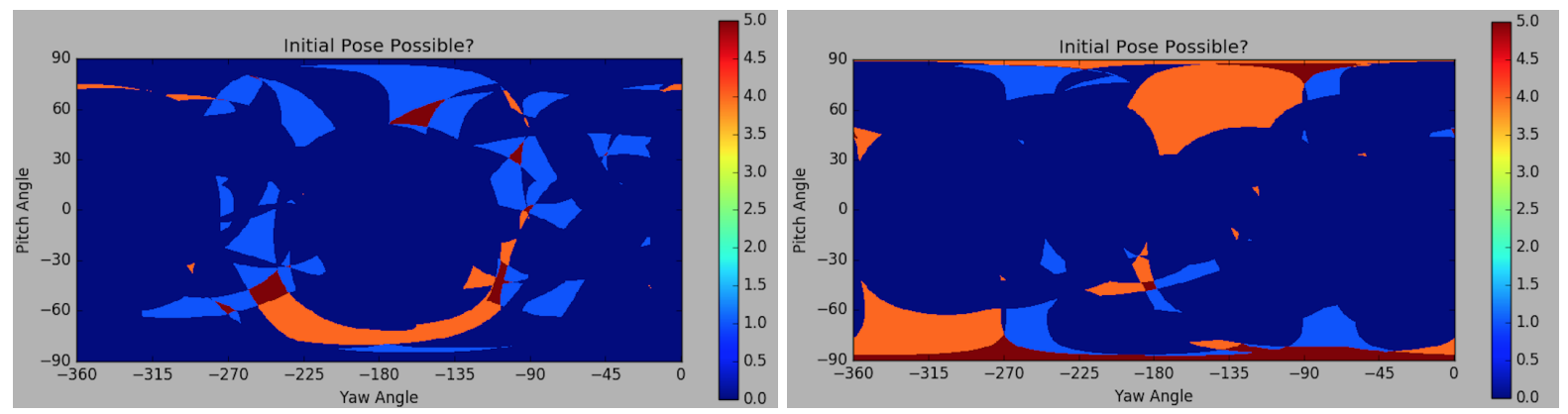

Figure 7. 2D Initial Pose Possible for the Vive Tracker (Left) and our model (Right)

The $3 D$ Initial Pose Possible view is shown in Figure 8. The simulation shows whether it is possible, or not, to acquire an initial pose from various positions. An initial pose is acquired only using the IR sensors -the IMUs alone will drift. Because of this, at least four sensors need to be visible from a position (one removed from the plane of the other three). Therefore, the plot can be used to highlight poses with co-planar sensors.
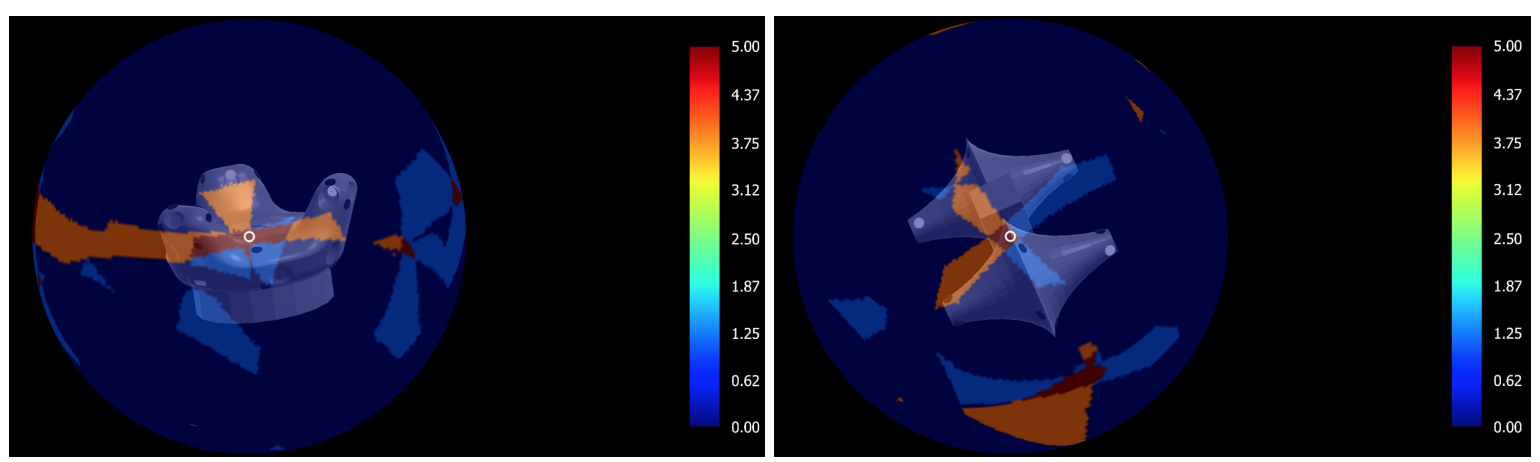

Figure 8. 3D Initial Pose Possible for the Vive Tracker (Left) and our model (Right)

The Final plot is the 2D Pose Translation Errors, shown in Figure 9, which highlights the factors which drive translation error. It demonstrates the foreshortening of sensor distance with translation away from Base Stations. For example, the outcome is "Green" when sensor distance shrinks, but 
remains significant; the outcome is "Red" when sensor distance is already marginal, but gets worse with translation.
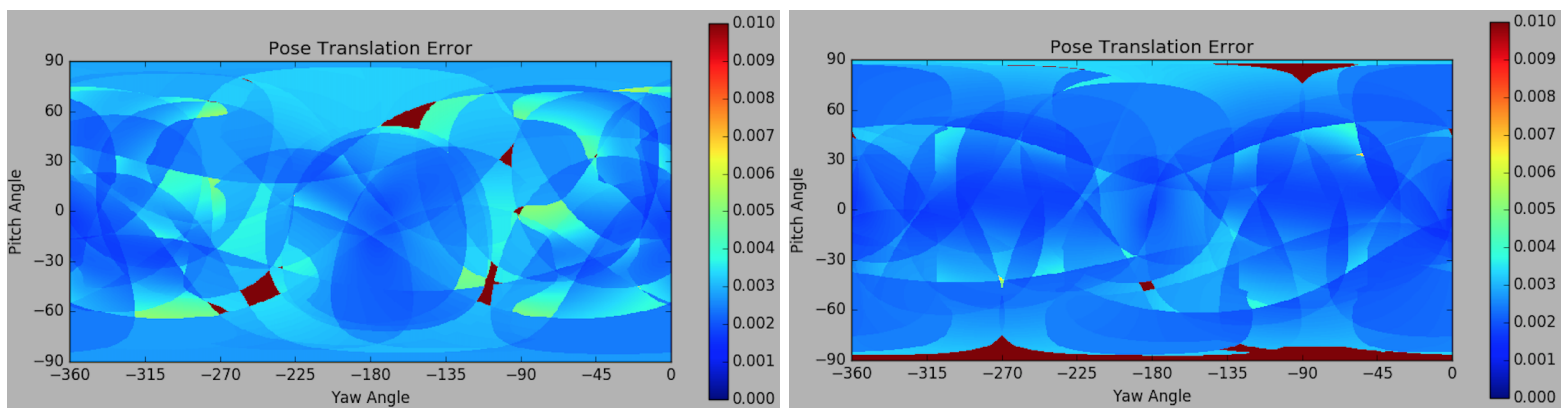

Figure 9. 2D Pose Translation Error for the Vive Tracker (Left) and our model (Right)

The 3D Pose Translation Error is shown in Figure 10. It is used to identify poses with insufficient baseline. If sensors are co-linear, problems with rotation error will also appear because sensors do not even have a plane. Then either of those errors will result in a failure to capture an initial pose. Additionally, if sensors are co-linear and are struck by the laser at specific times, there is no way of understanding the rotation and position from these co-linear sensors alone. Fixing this is simply pulling a sensor out of line (and out of plane as to avoid rotation errors), as demonstrated by the Tracker and our model.
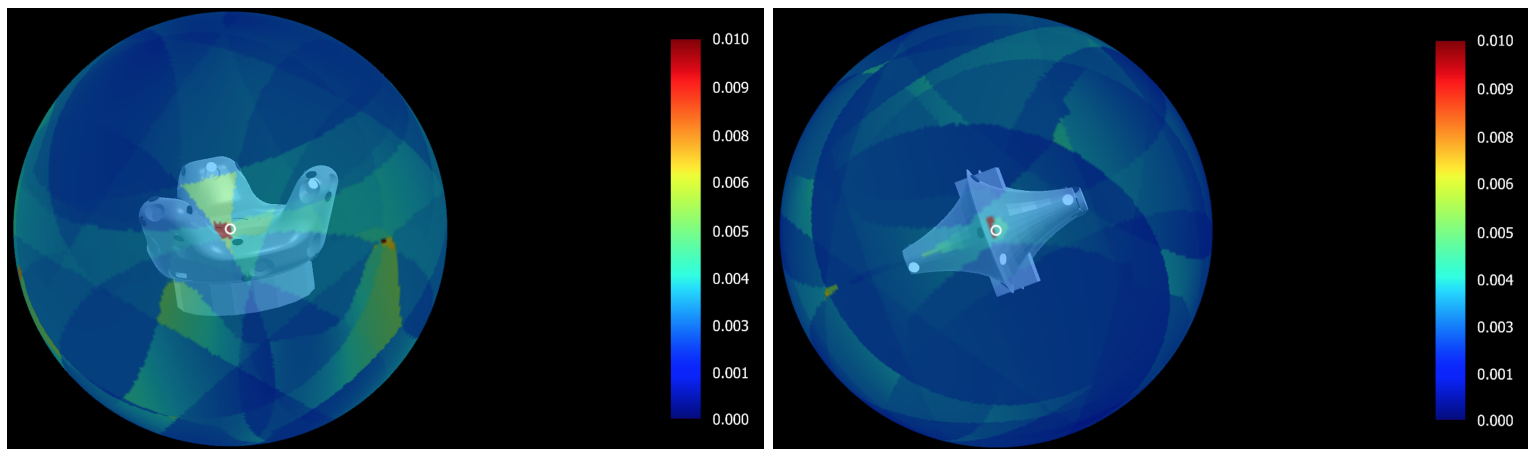

Figure 10. 3D Pose Translation Error for the Vive Tracker (Left) and our model (Right)

\section{CONCLUSION}

Our approach was to compare against the simulation results of a known good object (the Vive Tracker). The sensor placement simulation outputs on our model give better results than the Tracker; the Tracker seems to perform poorly (Red) when the (yaw angle, pitch angle) is $\left(-110^{\circ},-40^{\circ}\right),\left(-110^{\circ}, 40^{\circ}\right)$ or $\left(-250^{\circ},-40^{\circ}\right)$, while our model seems to perform poorly when the pitch angle is $-90^{\circ}$. The Tracker results might not be at its best, however; the sensors are placed/oriented slightly different on the physical product, as seen from these "dents (sensors covering)" on the casing surface. Thus, the performance of the Tracker, in real life final product, might be better than the simulation.

\subsection{Challenges and Limitations}

Although simulation output is a good way to gauge relative performance, it is not a substitute for prototyping the physical product and testing it in VR, which makes object design challenging:

1. On an industrial design level, consumer products need to look and feel great, as well as perform, especially VR products. This is challenging because appealing design features do not simply work just by themselves; for example, miniaturizing the model increases translation error, flattening the surfaces increases the rotation error and low-profiling tends to increase both translation and rotation error.

2. On a mechanical design level, facets on models show improved performance, but the number of available sides is usually limited. Multiple parts facilitates ejection, which allows for sensors to face multiple directions. Sensor covering requires many designs choices like finding an IR transmissive and diffusive material and applying that to a curved surfaces. 
3. On an electrical design level, it is challenging to have 24 sensors interconnected and distributed over the surface of the object since four wires are needed for each sensor. There are also circuit design challenges such as: circuit size and shape.

\subsection{Future Work}

Overall, the suggested model still performs relatively well based on the simulation outputs. Future research will involve prototyping the physical product and testing it in VR. Areas with poor performance will be inspected; there is a room for optimization when deciding on sensors covering and building the physical product.

\section{ACKNOWLEDGMENTS}

This project has received funding from the European Union's Horizon 2020 research and innovation programme under grant agreement No 856998. This research has been also partially financially supported by Academy of Finland 6Genesis Flagship (grant 318927) and the Nokia Foundation 2020 Scholarship (grant 20200091).

\section{REFERENCES}

Barhoush, Y., Georgiev, G.V. and Loudon, B. (2020), "Empathy and Idea Generation: Exploring the Design of a Virtual Reality Controller for Rehabilitation Purposes", Proceedings of the Sixth International Conference on Design Creativity (ICDC 2020), https://doi.org/10.35199/ICDC.2020.36.

Burdea, G., Kim, N., Polistico, K., Kadaru, A., Grampurohit, N., Roll, D. and Damiani, F. (2019), “Assistive game controller for artificial intelligence-enhanced telerehabilitation post-stroke", Assistive Technology, Vol. 0 No. 0, pp. 1-12, 10.1080/10400435.2019.1593260. Publisher: Taylor \& Francis_eprint: https://doi.org/10.1080/10400435.2019.1593260.

Capece, N., Erra, U. and Romaniello, G. (2018), “A Low-Cost Full Body Tracking System in Virtual Reality Based on Microsoft Kinect", in: L.T. De Paolis and P. Bourdot (Editors), Augmented Reality, Virtual Reality, and Computer Graphics, Lecture Notes in Computer Science, Springer International Publishing, Cham, pp. 623-635, 10.1007/978-3-319-95282-644:

Carulli, M., Bordegoni, M., Ferrise, F., Gallace, A., Gustafsson, M. and Pfuhl, T. (2018), "Simulating Multisensory Wine Tasting Experience", https://doi.org/10.21278/idc.2018.0485. Conference Name: DESIGN 2018 - 15th International Design Conference Pages: 2177-2186.

Du, Y.C., Shih, C.B., Fan, S.C., Lin, H.T. and Chen, P.J. (2018), “An IMU-compensated skeletal tracking system using Kinect for the upper limb”, Microsystem Technologies, Vol. 24 No. 10, pp. 4317-4327, 10.1007/s00542-018-3769-6.

Freire, R., Glowacki, B.R., Williams, R.R., Wonnacott, M., Jamieson-Binnie, A. and Glowacki, D.R. (2020), "OMG-VR: Open-source Mudra Gloves for Manipulating Molecular Simulations in VR", arXiv:1901.03532 [physics]. ArXiv: 1901.03532.

Gerschütz, B., Fechter, M., Schleich, B. and Wartzack, S. (2019), "A Review of Requirements and Approaches for Realistic Visual Perception in Virtual Reality", Proceedings of the Design Society: International Conference on Engineering Design, Vol. 1 No. 1, pp. 1893-1902, 10/gf6bpk. Publisher: Cambridge University Press.

Honnet, C. and Lopes, G. (2019), "HiveTracker: 3D positioning for ubiquitous embedded systems", in: Adjunct Proceedings of the 2019 ACM International Joint Conference on Pervasive and Ubiquitous Computing and Proceedings of the 2019 ACM International Symposium on Wearable Computers, UbiComp/ISWC '19 Adjunct, Association for Computing Machinery, New York, NY, USA, pp. 288-291, $10.1145 / 3341162.3349295$.

Horvat, N., Škec, S., Martinec, T., Lukačević, F. and Perišić, M.M. (2019), "Comparing Virtual Reality and Desktop Interface for Reviewing 3D CAD Models", Proceedings of the Design Society: International Conference on Engineering Design, Vol. 1 No. 1, pp. 1923-1932, 10/ghm594. Publisher: Cambridge University Press.

Kruk, E.v.d. and Reijne, M.M. (2018), “Accuracy of human motion capture systems for sport applications; state-of-the-art review", European Journal of Sport Science, Vol. 18 No. 6, pp. 806-819, 10.1080/17461391.2018.1463397. Publisher: Routledge_eprint: https://doi.org/10.1080/17461391.2018.1463397.

Murray, B.C.M., Peele, B.N., Xu, P., Spjut, J., Shapira, O., Luebke, D. and Shepherd, R.F. (2018), “A variable shape and variable stiffness controller for haptic virtual interactions", in: 2018 IEEE International Conference on Soft Robotics (RoboSoft), pp. 264-269, 10.1109/ROBOSOFT.2018.8404930. 
Naeemabadi, M.R., Dinesen, B.I., Andersen, O.K., Najafi, S. and Hansen, J. (2018), "Evaluating accuracy and usability of Microsoft Kinect sensors and wearable sensor for tele knee rehabilitation after knee operation", Proceedings of the 11th International Joint Conference on Biomedical Engineering Systems and Technologies, Biostec 2018; Biodevices 2018, 19-21 January 2018, Funchal, Madeira, Portugal, pp. 128-135, 10.5220/0006578201280135. Publisher: SCITEPRESS Digital Library.

Rahimi, A., Zhou, J. and Haghani, S. (2020), “A VR gun controller with Recoil Adjustability”, in: 2020 IEEE International Conference on Consumer Electronics (ICCE), pp. 1-2, 10.1109/ICCE46568.2020.9043008. ISSN: 2158-4001.

Shigeyama, J., Hashimoto, T., Yoshida, S., Narumi, T., Tanikawa, T. and Hirose, M. (2019), “Transcalibur: A Weight Shifting Virtual Reality Controller for 2D Shape Rendering based on Computational Perception Model", in: Proceedings of the 2019 CHI Conference on Human Factors in Computing Systems, CHI '19, Association for Computing Machinery, New York, NY, USA, pp. 1-11, 10.1145/3290605.3300241.

Sinclair, M., Ofek, E., Gonzalez-Franco, M. and Holz, C. (2019), "CapstanCrunch: A Haptic VR Controller with User-supplied Force Feedback”, in: Proceedings of the 32nd Annual ACM Symposium on User Interface Software and Technology, UIST '19, Association for Computing Machinery, New York, NY, USA, pp. 815-829, 10.1145/3332165.3347891.

Stevenson, J. (2019), "Bugles, trumpets, and beltrami”, Meditations on Mathematics, Vol. 24.

Suznjevic, M., Mandurov, M. and Matijasevic, M. (2017), "Performance and QoE assessment of HTC Vive and Oculus Rift for pick-and-place tasks in VR”, pp. 1-3, 10.1109/QoMEX.2017.7965679. ISSN: 2472-7814.

Tian, Y., Meng, X., Tao, D., Liu, D. and Feng, C. (2015), "Upper limb motion tracking with the integration of IMU and Kinect", Neurocomputing, Vol. 159, pp. 207-218, 10.1016/j.neucom.2015.01.071.

Tinguy, X.d., Howard, T., Pacchierotti, C., Marchal, M. and Lécuyer, A. (2020), "WeATaViX: WEarable Actuated TAngibles for VIrtual reality eXperiences", Vol. 12272, pp. 297-306.

Whitmire, E., Benko, H., Holz, C., Ofek, E. and Sinclair, M. (2018), "Haptic Revolver: Touch, Shear, Texture, and Shape Rendering on a Reconfigurable Virtual Reality Controller", in: Proceedings of the 2018 CHI Conference on Human Factors in Computing Systems, CHI '18, Association for Computing Machinery, New York, NY, USA, pp. 1-12, 10.1145/3173574.3173660.

Wittmann, F., Lambercy, O. and Gassert, R. (2019), "Magnetometer-Based Drift Correction During Rest in IMU Arm Motion Tracking", Sensors, Vol. 19 No. 6, p. 1312, 10.3390/s19061312. Number: 6 Publisher: Multidisciplinary Digital Publishing Institute.

Yi, H., Hong, J., Kim, H. and Lee, W. (2019), "DexController : Designing a VR Controller with GraspRecognition for Enriching Natural Game Experience", in: 25th ACM Symposium on Virtual Reality Software and Technology, VRST '19, Association for Computing Machinery, New York, NY, USA, pp. $1-11,10.1145 / 3359996.3364263$.

Zenner, A., Degraen, D., Daiber, F. and Krüger, A. (2020), "Demonstration of Drag:on - A VR Controller Providing Haptic Feedback Based on Drag and Weight Shift", in: Extended Abstracts of the 2020 CHI Conference on Human Factors in Computing Systems, CHI EA '20, Association for Computing Machinery, New York, NY, USA, pp. 1-4, 10.1145/3334480.3383145. 